\title{
Pulmonary Artery
}

National Cancer Institute

\section{Source}

National Cancer Institute. Pulmonary Artery. NCI Thesaurus. Code C12774.

Either one of two arteries arising from the pulmonary trunk that carry deoxygenated blood to the lungs. 